\title{
FOURIER RESTRICTION TO CONVEX SURFACES OF REVOLUTION IN $\mathbb{R}^{3}$
}

\author{
Faruk Abi-Khuzam and Bassam Shayya
}

\begin{abstract}
If $\Gamma$ is a $C^{3}$ hypersurface in $\mathbb{R}^{n}$ and $d \sigma$ is induced Lebesgue measure on $\Gamma$, then it is well known that a Tomas-Stein Fourier restriction estimate on $\Gamma$ implies that $\Gamma$ has a nowhere vanishing Gaussian curvature. In a recent paper, Carbery and Ziesler observed that if induced Lebesgue measure is replaced by affine surface area, then a Tomas-Stein restriction estimate on $\Gamma$ implies that $\Gamma$ satisfies the affine isoperimetric inequality. Since the only property needed for a hypersurface to satisfy the affine isoperimetric inequality is convexity, this raised the question of whether a TomasStein restriction estimate can be obtained for flat but convex hypersurfaces in $\mathbb{R}^{n}$ such as $\Gamma(x)=\left(x, e^{-1 /|x|^{m}}\right), m=1,2, \ldots$ We prove that this is indeed the case in dimension $n=3$.
\end{abstract}

\section{Introduction}

Let $\Gamma$ be a $C^{3}$ hypersurface in $\mathbb{R}^{n}$ and $d \sigma$ a measure on $\Gamma$. A TomasStein Fourier restriction estimate for the pair $(\Gamma, d \sigma)$ is an inequality of the form

$$
\|\widehat{f}\|_{L^{2}(d \sigma)} \lesssim\|f\|_{L^{\frac{2 n+2}{n+3}\left(\mathbb{R}^{n}\right)}}
$$

for $f \in C_{0}\left(\mathbb{R}^{n}\right)$.

The existence of restriction estimates such as (1), as well as their connection with the geometry of $\Gamma$, or with the decay of the Fourier transform of $d \sigma$, has been a subject of great interest. See [9, pp. 368-373] for some important applications of these estimates.

The choice of the measure $d \sigma$ is not completely arbitrary. It usually reflects some aspect of the geometry of $\Gamma$. Two important choices of $d \sigma$ are induced Lebesgue measure and affine surface area. In the former case, if $\Gamma$ is assumed to have non-vanishing Gaussian curvature, (1) is a

2000 Mathematics Subject Classification. 42B10, 42B15.

Key words. Fourier transform, restriction, affine surface area. 
classical result of Tomas and Stein (see [10] and [9]). Conversely, if (1) holds with induced Lebesgue measure, then a result of Iosevich and Lu [3] (see also [2]), implies that $\Gamma$ has non-vanishing Gaussian curvature. The proof of this converse uses, among other things, a Knapp-type scaling argument. To see how this argument goes, consider the special case where $\Gamma$ is a surface of revolution given by $\Gamma(x)=(x, \phi(x))$, where $\phi(x)=\gamma(|x|)$, and $\gamma:[0, b) \rightarrow \mathbb{R}$ is increasing and satisfies $\gamma(0)=\gamma^{\prime}(0)=0$. For $0<\delta<b$, let $S_{\delta}=\left\{(x, \gamma(|x|):|x| \leq \delta\}\right.$ and let $f_{\delta}$ be a smoothedout characteristic function of $S_{\delta}$. It is then easy to see that $\left\|f_{\delta}\right\|_{L^{2}(d \sigma)} \lesssim$ $\delta^{(n-1) / 2}$, and that $\left|\widehat{f_{\delta} d \sigma}\right| \gtrsim \delta^{n-1}$ on a $(C / \delta) \times \cdots \times(C / \delta) \times(C / \gamma(\delta))$ box in $\mathbb{R}^{n}$ (for a suitable constant $C$ ). Now if (1) holds then, by duality, the equivalent adjoint restriction estimate

$$
\|\widehat{f d \sigma}\|_{L^{\frac{2 n+2}{n-1}\left(\mathbb{R}^{n}\right)}} \lesssim\|f\|_{L^{2}(d \sigma)}
$$

also holds. Applying (2) to $f_{\delta}$ we obtain

$$
\delta^{2} \lesssim \gamma(\delta)
$$

and this implies that $\gamma^{\prime \prime}(0) \neq 0$. In particular $\gamma$ cannot have vanishing Gaussian curvature at the origin. A more elaborate argument shows that the same conclusion holds in general.

In the latter case, say when $\Gamma(x)=(x, \phi(x))$, the affine surface area on $\Gamma$ is given as the pushforward under $\Gamma$ of the $(n-1)$-dimensional measure $\left|K_{\phi}(x)\right|^{1 /(n+1)} d x$, where $K_{\phi}(x)=\operatorname{det}(\operatorname{Hess} \phi(x))$ is the affine curvature of $\Gamma$. To see what kind of geometry on $\Gamma$ may be expected, take the case of a surface of revolution considered above. The radial assumption on $\phi$, e.g. $\phi(x)=\gamma(|x|)$, simplifies matters and one computes that

$$
K_{\phi}(x)=\gamma^{\prime \prime}(|x|)\left(\frac{\gamma^{\prime}(|x|)}{|x|}\right)^{n-2} .
$$

If we then take $d \sigma$ in the adjoint restriction estimate (2), which is equivalent to (1), to be affine surface area and use the function $f_{\delta}$ in it, we arrive $[\mathbf{1}]$ at the inequality

$$
\int_{0}^{\delta}\left|\gamma^{\prime \prime}(r)\left(\frac{\gamma^{\prime}(r)}{r}\right)^{n-2}\right|^{1 /(n+1)} r^{n-2} d r \lesssim\left(\delta^{n-1} \gamma(\delta)\right)^{(n-1) /(n+1)} .
$$

But now this inequality does not imply non-vanishing curvature. Rather, it is satisfied by any convex $\gamma$, regardless of how flat it is at the origin, e.g. it is satisfied by $\gamma(t)=e^{-1 / t^{m}}, m$ any positive integer. In fact, even if $\phi$ is not radial, there is a similar scaling argument that can be applied, and it leads to the conclusion that $\phi$ satisfies the affine isoperimetric 
inequality of affine differential geometry, which is certainly true whenever $\phi$ is convex. For more details we refer the reader to [1, pp. 409-410], [5, Chapter 5], and [6].

An earlier result of Sjölin [8] had already established that, if the dimension $n=2$, and $\phi$ is convex, then the restriction inequality holds true for affine surface area. The strength of this result, along with the above considerations, suggested that, perhaps, the geometric condition of convexity of $\phi$ could imply a restriction result for affine surface area in higher dimensions. But if only convexity is to be used, functions such as $\phi(x)=e^{-1 /|x|^{m}}$ have to be admitted. In attempting to prove this result, i.e. to show that convexity implies restriction, Carbery and Ziesler [1] considered the implications of a decay assumption on the Fourier transform of $d \sigma$.

Kenig, Ponce and Vega [4] proved that if the decay assumption

$$
\left.\left|\int_{B(0, b)} e^{-2 \pi i \xi \cdot \Gamma(x)}\right| K_{\phi}(x)\right|^{\frac{1}{2}+i \alpha} d x \mid \lesssim \frac{(1+|\alpha|)^{N}}{\left|\xi_{n}\right|}
$$

was true for all real $\alpha$ and some integer $N$, then (2) holds ${ }^{1}$. When testing (4) on $\phi(x)=e^{-1 /|x|^{m}}$, Carbery and Ziesler [1] found that it did not hold true in dimension $n=3$. This, of course, did not mean that there was no restriction result for $\phi(x)=e^{-1 /|x|^{m}}$. More recently, the same restriction question was addressed in [7]. A consequence of the results there implies that if $\phi(\cdot)=\gamma(|\cdot|)$, where $\gamma$ is convex, $\gamma(0)=$ $\gamma^{\prime}(0)=0, \gamma^{(3)}(t)$ non-negative, and if

$$
\sup _{0<t<b} \frac{t \gamma^{\prime \prime}(t)}{\gamma^{\prime}(t)} \leq C<\infty,
$$

then the restriction estimate (1) holds for affine surface area in dimension $n=3$. Testing this last condition on $\gamma(t)=e^{-1 / t^{m}}$, where $\left.0<t<b_{m}, b_{m}=m /(3 m+3)\right)$, one finds that

$$
\sup _{0<t<b_{m}} \frac{t \gamma^{\prime \prime}(t)}{\gamma^{\prime}(t)}=\sup _{0<t<b_{m}}\left(\frac{m}{t^{m}}-m-1\right)=\infty .
$$

Once again, the function $e^{-1 / t^{m}}$ was precluded from the result.

It turns out that, at least for surfaces of revolution $\Gamma(x)=(x, \phi(x))$, $\phi(x)=\gamma(|x|)$, a Tomas-Stein restriction estimate for affine surface area

\footnotetext{
${ }^{1}$ This connection between decay and restriction is valid in dimensions $n=2,3$. In dimensions $n \geq 4$, one has to modify things slightly by inserting a smooth cut-off function into both (2) and (4), see [1] for further details.
} 
does hold in the presence of convexity, if we add the condition that

$$
\sup _{0<t<b} \frac{\gamma(t) \gamma^{\prime \prime}(t)}{\gamma^{\prime}(t)^{2}} \leq C<\infty \text {. }
$$

Now testing this condition on $\gamma(t)=e^{-1 / t^{m}}$ one finds that

$$
\sup _{0<t<b_{m}} \frac{\gamma(t) \gamma^{\prime \prime}(t)}{\gamma^{\prime}(t)^{2}}=\sup _{0<t<b_{m}}\left(1-\frac{m+1}{m} t^{m}\right) \leq 1 .
$$

We thus have a Tomas-Stein restriction result that includes the surfaces $\Gamma(x)=\left(x, e^{-1 /|x|^{m}}\right)$ in $\mathbb{R}^{3}$.

The purpose of this paper is to obtain restriction estimates for convex surfaces of revolution in $\mathbb{R}^{3}$. A major role is played by the function

$$
\frac{\gamma(t) \gamma^{\prime \prime}(t)}{\gamma^{\prime}(t)^{2}}
$$

and our results only require the boundedness of certain $L^{p_{0}}$ norms of this function. In particular, we obtain a Tomas-Stein restriction estimate for surfaces of revolution in $\mathbb{R}^{3}$ satisfying (5). We find it useful to prove our results in a little more general setting. In Section 2 we introduce a family of measures $d \sigma_{\gamma}$, state a general $\left(L^{p}, L^{q}\right)$ restriction result for such measures, and obtain as a corollary the result on $\Gamma(x)=\left(x, e^{-1 /|x|^{m}}\right)$. In Section 3 we present the main component of our proof. In Section 4 we prove our results.

\section{Statement of results}

Let $0<b \leq \infty$, and denote by $B(0, b)$ the ball in $\mathbb{R}^{2}$ of center 0 and radius $b$. Let $\mathcal{C}([0, b))$ be the set of all real-valued functions $\gamma \in C^{3}([0, b))$ such that $\gamma(0)=\gamma^{\prime}(0)=0, \gamma^{\prime \prime}(t)>0$ for $0<t<b$, and $\gamma^{(3)}(t) \geq 0$ for $0 \leq t<b$.

Suppose $0 \leq \lambda \leq 1,1 \leq p, p_{0} \leq \infty, 4 \leq q \leq \infty$, and $1 / p+2 / q \leq 1$. For $\gamma \in \mathcal{C}([0, b))$, let $d \sigma_{\gamma}$ be the pushforward under the map $x \rightarrow$ $(x, \gamma(|x|)$ of the two-dimensional measure

$$
\left(\frac{\gamma^{\prime}(|x|)^{3-2 \lambda} \gamma^{\prime \prime}(|x|)^{\lambda}}{|x| \gamma(|x|)^{1-\lambda}}\right)^{\frac{p^{\prime}}{2 q}} d x
$$

with the understanding that when $p^{\prime}=q=\infty, p^{\prime} /(2 q)$ is set to be equal to $1 / 4$; so that $p^{\prime} /(2 q)=1 / 4$ on the sharp line $1 / p+2 / q=1$ including the point $(1 / p, 1 / q)=(1,0)$. 
Theorem 1. If $1 / p+2 / q=1-1 / p_{0}$, then

(8) $\left\|\widehat{f d \sigma_{\gamma}}\right\|_{L^{q}\left(\mathbb{R}^{3}\right)} \leq C_{q}\left\|\left(\frac{\gamma(|\cdot|) \gamma^{\prime \prime}(|\cdot|)}{\gamma^{\prime}(|\cdot|)^{2}}\right)^{\frac{\lambda}{2 q}}\right\|_{L^{p_{0}}(B(0, b))}\|f\|_{L^{p}\left(d \sigma_{\gamma}\right)}$

for all $(f, \gamma) \in C_{0}\left(\mathbb{R}^{3}\right) \times \mathcal{C}([0, b))$, where $C_{q}=4\left(2^{7 / 6} \pi\right)^{3 /(2 q)}$.

Notice that if $\lambda=1$, then the density of the measure (7) is $\left|K_{\gamma(|\cdot|)}(x)\right|^{p^{\prime} /(2 q)}$, so if in addition $1 / p+2 / q=1$, then $d \sigma_{\gamma}$ is the same affine surface area measure we described in Section 1.

Corollary 1. Suppose $\gamma \in \mathcal{C}([0, b))$ is such that

$$
\left\|\left(\frac{\gamma(|\cdot|) \gamma^{\prime \prime}(|\cdot|)}{\gamma^{\prime}(|\cdot|)^{2}}\right)^{\frac{1}{2 q}}\right\|_{L^{p_{0}}(B(0, b))}<\infty .
$$

Let $\lambda=1$ and $d \sigma=d \sigma_{\gamma}$. If $1 / p+2 / q=1-1 / p_{0}$, then

$$
\|\widehat{f d \sigma}\|_{L^{q}\left(\mathbb{R}^{3}\right)} \lesssim\|f\|_{L^{p}(d \sigma)}
$$

for all $f \in L^{p}(d \sigma)$.

For example if $\gamma(t)=e^{-1 / t^{m}}$, then by $(6)$,

$$
\left\|\left(\frac{\gamma(|\cdot|) \gamma^{\prime \prime}(|\cdot|)}{\gamma^{\prime}(|\cdot|)^{2}}\right)^{\frac{1}{2 q}}\right\|_{L^{p_{0}\left(B\left(0, b_{m}\right)\right)}} \leq\left(\pi b_{m}^{2}\right)^{1 / p_{0}}<\infty
$$

for $1 \leq p_{0} \leq \infty$, and so the adjoint restriction estimate in Corollary 1 holds for $\gamma(t)=e^{-1 / t^{m}}$ whenever $4 \leq q \leq \infty$ and $1 / p+2 / q \leq 1$.

If, as another example, we take $\gamma(t)=-t \log (1-t)$, which is in $\mathcal{C}([0,1))$, then

$$
\left\|\left(\frac{\gamma(|\cdot|) \gamma^{\prime \prime}(|\cdot|)}{\gamma^{\prime}(|\cdot|)^{2}}\right)^{\frac{1}{2 q}}\right\|_{L^{p_{0}}(B(0,1))} \approx\left\|\left(-\frac{\log (1-|\cdot|)}{|\cdot|}\right)^{\frac{1}{2 q}}\right\|_{L^{p_{0}}(B(0,1))}
$$

is finite for $1 \leq p_{0}<\infty$ but not for $p_{0}=\infty$ (except if $q=\infty$ ), and so the adjoint restriction estimate in Corollary 1 holds for $\gamma(t)=-t \log (1-t)$ whenever $4 \leq q \leq \infty$ and $1 / p+2 / q<1$.

\section{Main estimate}

Let $\tilde{B}=B(0, b) \cap\left\{x=\left(x_{1}, x_{2}\right) \in \mathbb{R}^{2}: x_{1}, x_{2}>0\right\}$. The purpose of this section is to prove the following proposition. 
Proposition 1. Suppose $0<b \leq \infty$ and $\gamma \in \mathcal{C}([0, b))$. Then

$$
\begin{aligned}
& \int_{\tilde{B}} \int_{\tilde{B}} h(u+v, \gamma(|u|)+\gamma(|v|))\left(\frac{\gamma^{\prime}(|u|)^{3}}{|u| \gamma(|u|)} \frac{\gamma^{\prime}(|v|)^{3}}{|v| \gamma(|v|)}\right)^{\frac{1}{4}} d u d v \\
& \leq\left(2^{7 / 6} \pi\right)^{3 / 2}\|h\|_{L^{1}\left(\mathbb{R}^{3}\right)}
\end{aligned}
$$

for all Lebesgue measurable $h: \mathbb{R}^{3} \rightarrow[0, \infty]$.

Proof: Denoting the integral on the left-hand side of the inequality by I, and changing into polar coordinates, we have

$\mathrm{I}=\int_{0}^{b} \int_{0}^{b} \int_{0}^{\frac{\pi}{2}} \int_{0}^{\frac{\pi}{2}} h\left(r e^{i \theta}+s e^{i \phi}, \gamma(r)+\gamma(s)\right) d \theta d \phi\left(\frac{r^{3} \gamma^{\prime}(r)^{3} s^{3} \gamma^{\prime}(s)^{3}}{\gamma(r) \gamma(s)}\right)^{\frac{1}{4}} d r d s$

The change of variable $x=r e^{i \theta}+s e^{i \phi}(\operatorname{cf}[7])$ shows that

$$
\begin{aligned}
\int_{0}^{\frac{\pi}{2}} \int_{0}^{\theta} h & \left(r e^{i \theta}+s e^{i \phi}, \gamma(r)+\gamma(s)\right) d \phi d \theta \\
& \leq \int_{\sqrt{r^{2}+s^{2}}<|x|<r+s} \frac{2 h(x, \gamma(r)+\gamma(s))}{\sqrt{\left(|x|^{2}-(r-s)^{2}\right)\left((r+s)^{2}-|x|^{2}\right)}} d x
\end{aligned}
$$

So

$$
\begin{aligned}
\int_{0}^{\frac{\pi}{2}} & \int_{0}^{\frac{\pi}{2}} h\left(r e^{i \theta}+s e^{i \phi}, \gamma(r)+\gamma(s)\right) d \phi d \theta \\
& \leq \int_{\sqrt{r^{2}+s^{2}}<|x|<r+s} \frac{4 h(x, \gamma(r)+\gamma(s))}{\sqrt{\left(|x|^{2}-(r-s)^{2}\right)\left((r+s)^{2}-|x|^{2}\right)}} d x \\
& \leq \int_{\sqrt{r^{2}+s^{2}}<|x|<r+s} \frac{4 h(x, \gamma(r)+\gamma(s))}{\sqrt{(2 r s)\left((r+s)^{2}-|x|^{2}\right)}} d x \\
& \leq \int_{|x|<r+s} \frac{2 h(x, \gamma(r)+\gamma(s))}{(r s)^{\frac{3}{4}} \sqrt{r+s-|x|}} d x
\end{aligned}
$$


where we have used the inequality $r+s \geq 2 \sqrt{r s}$. It follows that

$$
\begin{aligned}
\mathrm{I} & \leq 2 \int_{0}^{b} \int_{0}^{b} \int_{|x|<r+s} \frac{h(x, \gamma(r)+\gamma(s))}{\sqrt{r+s-|x|}} d x\left(\frac{\gamma^{\prime}(r)^{3} \gamma^{\prime}(s)^{3}}{\gamma(r) \gamma(s)}\right)^{\frac{1}{4}} d r d s \\
& =2 \int_{B(0,2 b)} \int_{0}^{b} \int_{0}^{b} h(x, \gamma(r)+\gamma(s)) \frac{\chi_{E}(r, s)}{\sqrt{r+s-x}}\left(\frac{\gamma^{\prime}(r)^{3} \gamma^{\prime}(s)^{3}}{\gamma(r) \gamma(s)}\right)^{\frac{1}{4}} d r d s d x \\
& =4 \int_{B(0,2 b)} \int_{0}^{b} \int_{0}^{b} h(x, \gamma(r)+\gamma(s)) \frac{\chi_{F}(r, s)}{\sqrt{r+s-x}}\left(\frac{\gamma^{\prime}(r)^{3} \gamma^{\prime}(s)^{3}}{\gamma(r) \gamma(s)}\right)^{\frac{1}{4}} d r d s d x \\
& =4 \int_{B(0,2 b)} \mathrm{II} d x,
\end{aligned}
$$

where $E=\{(r, s) \in(0, b) \times(0, b): r+s>|x|\}, F=\{(r, s) \in E: s<r\}$, and

$$
\mathrm{II}=\int_{0}^{b} \int_{0}^{b} h(x, \gamma(r)+\gamma(s)) \frac{\chi_{F}(r, s)}{\sqrt{r+s-x}}\left(\frac{\gamma^{\prime}(r)^{3} \gamma^{\prime}(s)^{3}}{\gamma(r) \gamma(s)}\right)^{\frac{1}{4}} d r d s .
$$

To estimate II, we shall first apply the change of variable

$$
\begin{aligned}
& r=r(t, y)=\gamma^{-1}\left(y \sin ^{2} t\right) \\
& s=s(t, y)=\gamma^{-1}\left(y \cos ^{2} t\right),
\end{aligned}
$$

which is defined on the open set

$$
\Omega=\left\{(t, y) \in \mathbb{R}^{2}: \frac{\pi}{4}<t<\frac{\pi}{2}, y>0\right\}
$$

so, with a slight abuse of notation, $(r, s)$ is now a mapping from $\Omega$ to $\mathbb{R}^{2}$. The Jacobian of this mapping is

$$
J_{(r, s)}(t, y)=\frac{2 y \sin t \cos ^{3} t+2 y \sin ^{3} t \cos t}{\gamma^{\prime}\left(\gamma^{-1}\left(y \sin ^{2} t\right)\right) \gamma^{\prime}\left(\gamma^{-1}\left(y \cos ^{2} t\right)\right)}=\frac{y \sin 2 t}{\gamma^{\prime}(r) \gamma^{\prime}(s)} .
$$

But $^{2}$

$$
\gamma(r)=y \sin ^{2} t \quad \text { and } \quad \gamma(s)=y \cos ^{2} t
$$

so

$$
\gamma^{\prime}(r) \frac{\partial r}{\partial t}=y \sin 2 t \quad \text { and } \quad \gamma^{\prime}(s) \frac{\partial s}{\partial t}=-y \sin 2 t
$$

${ }^{2}$ To simplify the notation, we are writing $r, s, \partial r / \partial t$, and $\partial s / \partial t$ for $r(t, y), s(t, y)$, $\partial r / \partial t(t, y)$, and $\partial s / \partial t(t, y)$ respectively. 
and so

$$
y \sin 2 t=\sqrt{\gamma^{\prime}(r) \gamma^{\prime}(s)} \sqrt{\frac{\partial r}{\partial t}\left|\frac{\partial s}{\partial t}\right|}
$$

Thus

$$
J_{(r, s)}(t, y)=\frac{1}{\sqrt{\gamma^{\prime}(r) \gamma^{\prime}(s)}} \sqrt{\frac{\partial r}{\partial t}\left|\frac{\partial s}{\partial t}\right|}
$$

But also

$$
\frac{\gamma^{\prime}(r) \gamma^{\prime}(s)}{\gamma(r) \gamma(s)} \frac{\partial r}{\partial t}\left|\frac{\partial s}{\partial t}\right|=\frac{y^{2} \sin ^{2} 2 t}{\left(y \sin ^{2} t\right)\left(y \cos ^{2} t\right)}=4
$$

SO

$$
\left(\frac{\gamma^{\prime}(r)^{3} \gamma^{\prime}(s)^{3}}{\gamma(r) \gamma(s)}\right)^{\frac{1}{4}} J_{(r, s)}(t, y)=\left(4 \frac{\partial r}{\partial t}\left|\frac{\partial s}{\partial t}\right|\right)^{\frac{1}{4}}
$$

Next, to determine the domain of integration in the $t y$-plane, we make the following observations. By the convexity of $\gamma, \gamma(r)+\gamma(|x|-r)$, as a function of $r$, increases on the interval $(|x| / 2,|x|)$. So

$$
2 \gamma\left(\frac{|x|}{2}\right) \leq \gamma(r)+\gamma(|x|-r)<\gamma(r)+\gamma(s)
$$

whenever $|x| / 2<r<|x|$ and $|x|-r<s$, which are in turn satisfied whenever $s<r<|x|<r+s$. Also by the convexity of $\gamma$,

$$
2 \gamma\left(\frac{|x|}{2}\right) \leq \gamma(|x|) \leq \gamma(r)<\gamma(r)+\gamma(s)
$$

whenever $r \geq|x|$ and $s>0$. Thus

$$
2 \gamma\left(\frac{|x|}{2}\right)<\gamma(r)+\gamma(s)<2 \gamma(b)
$$

whenever $0<s<r<b$ and $|x|<r+s$. But, by the definition of the mapping $(r, s)$,

$$
y=\gamma(r)+\gamma(s)
$$

for all $(t, y) \in \Omega$, so

$$
2 \gamma\left(\frac{|x|}{2}\right)<y<2 \gamma(b)
$$

whenever $0<s<r<b$ and $|x|<r+s$. For any such (fixed) $y$, the range of $(r, s)$ is a curve in $\mathbb{R}^{2}$ that "enters" the closure of the domain 
of integration of II when $t=\pi / 4$ (i.e. when $s=r$ ) and "leaves" when $t=\tau(y)$ for some $\tau(y) \in(\pi / 4, \pi / 2]$. Thus

$$
\begin{aligned}
\mathrm{II} & =\int_{2 \gamma\left(\frac{|x|}{2}\right)}^{2 \gamma(b)} \int_{\frac{\pi}{4}}^{\tau(y)} h(x, y) \frac{1}{\sqrt{r+s-|x|}}\left(4 \frac{\partial r}{\partial t}\left|\frac{\partial s}{\partial t}\right|\right)^{\frac{1}{4}} d t d y \\
& =\int_{2 \gamma\left(\frac{|x|}{2}\right)}^{2 \gamma(b)} h(x, y) \int_{\frac{\pi}{4}}^{\tau(y)} \frac{\sqrt{2}}{\sqrt{r+s-|x|}}\left(\frac{\partial r}{\partial t}\left|\frac{\partial s}{\partial t}\right|\right)^{\frac{1}{4}} d t d y .
\end{aligned}
$$

Now, by the definition of $\tau(y)$,

$$
r+s=r(t, y)+s(t, y) \geq|x| \text { for } \frac{\pi}{4} \leq t \leq \tau(y),
$$

so, in particular,

$$
r(\tau(y), y)+s(\tau(y), y) \geq|x|
$$

and hence

$$
r+s-|x| \geq r+s-(r(\tau(y), y)+s(\tau(y), y)) \quad \text { for } \quad \frac{\pi}{4}<t<\tau(y) .
$$

Thus

$\mathrm{II} \leq \int_{2 \gamma\left(\frac{|x|}{2}\right)}^{2 \gamma(b)} h(x, y) \int_{\frac{\pi}{4}}^{\tau(y)} \frac{\sqrt{2}}{\sqrt{r+s-r(\tau(y), y)-s(\tau(y), y)}}\left(\frac{\partial r}{\partial t}\left|\frac{\partial s}{\partial t}\right|\right)^{\frac{1}{4}} d t d y$.

The rest of the proof will be devoted to estimating

$$
\frac{1}{\sqrt{r+s-r(\tau(y), y)-s(\tau(y), y)}}\left(\frac{\partial r}{\partial t}\left|\frac{\partial s}{\partial t}\right|\right)^{\frac{1}{4}}
$$

for $2 \gamma(|x| / 2)<y<2 \gamma(b)$ and $\pi / 4<t<\tau(y)$.

We start by examining the function $\partial r / \partial t+\partial s / \partial t$. By (10),

$$
\frac{\partial r}{\partial t}+\frac{\partial s}{\partial t}=\frac{y \sin 2 t}{\gamma^{\prime}(r)}-\frac{y \sin 2 t}{\gamma^{\prime}(s)}
$$

is negative for $\pi / 4<t<\pi / 2$ (since $\left.\gamma^{\prime}(s)<\gamma^{\prime}(r)\right)$, so

$$
\begin{aligned}
\left|\frac{\partial r}{\partial t}+\frac{\partial s}{\partial t}\right| & =\frac{y \sin 2 t}{\gamma^{\prime}(s)}-\frac{y \sin 2 t}{\gamma^{\prime}(r)} \\
& =2 y\left(\frac{\cos t}{\gamma^{\prime}(s)} \sin t-\frac{\sin t}{\gamma^{\prime}(r)} \cos t\right) \\
& =2 y \sqrt{\frac{\cos ^{2} t}{\gamma^{\prime}(s)^{2}}+\frac{\sin ^{2} t}{\gamma^{\prime}(r)^{2}}} \sin (t-\phi),
\end{aligned}
$$


where $\phi=\phi(t)$ is defined by

$$
\sin \phi=\frac{(\sin t) / \gamma^{\prime}(r)}{\sqrt{\frac{\cos ^{2} t}{\gamma^{\prime}(s)^{2}}+\frac{\sin ^{2} t}{\gamma^{\prime}(r)^{2}}}}, \quad \cos \phi=\frac{(\cos t) / \gamma^{\prime}(s)}{\sqrt{\frac{\cos ^{2} t}{\gamma^{\prime}(s)^{2}}+\frac{\sin ^{2} t}{\gamma^{\prime}(r)^{2}}}} .
$$

We shall need precise information about $\phi$ and $\partial^{2} r / \partial t^{2}+\partial^{2} s / \partial t^{2}$. For this we need the following easy, but important, observation. By integration by parts,

$$
\int_{0}^{\rho} 2 \gamma^{\prime}(\alpha) \gamma^{\prime \prime}(\alpha) d \alpha=2 \gamma(\rho) \gamma^{\prime \prime}(\rho)-2 \int_{0}^{\rho} \gamma(\alpha) \gamma^{(3)}(\alpha) d \alpha
$$

for $0<\rho<b$, and since $\gamma^{(3)}$ is nonnegative, we get

$$
\gamma^{\prime}(\rho)^{2} \leq 2 \gamma(\rho) \gamma^{\prime \prime}(\rho) \text { for } 0<\rho<b .
$$

(This is the only place where we use the assumptions that $\gamma$ is $C^{3}$ and $\gamma^{(3)}$ is nonnegative; everywhere else we need only require of $\gamma$ to be $C^{2}$ and convex.)

Differentiating both sides of (10) with respect to $t$, we have

$$
\gamma^{\prime \prime}(r)\left(\frac{\partial r}{\partial t}\right)^{2}+\gamma^{\prime}(r) \frac{\partial^{2} r}{\partial t^{2}}=2 y \cos 2 t
$$

and

$$
\gamma^{\prime \prime}(s)\left(\frac{\partial s}{\partial t}\right)^{2}+\gamma^{\prime}(s) \frac{\partial^{2} s}{\partial t^{2}}=-2 y \cos 2 t .
$$

This combined with (11) gives

$$
\frac{\gamma^{\prime}(r)^{2}}{2 \gamma(r)}\left(\frac{\partial r}{\partial t}\right)^{2}+\gamma^{\prime}(r) \frac{\partial^{2} r}{\partial t^{2}} \leq 2 y \cos 2 t
$$

and

$$
\frac{\gamma^{\prime}(s)^{2}}{2 \gamma(s)}\left(\frac{\partial s}{\partial t}\right)^{2}+\gamma^{\prime}(s) \frac{\partial^{2} s}{\partial t^{2}} \leq-2 y \cos 2 t
$$

But by (9) and (10),

$$
\frac{\gamma^{\prime}(r)^{2}}{2 \gamma(r)}\left(\frac{\partial r}{\partial t}\right)^{2}=2 y \cos ^{2} t \quad \text { and } \quad \frac{\gamma^{\prime}(s)^{2}}{2 \gamma(s)}\left(\frac{\partial s}{\partial t}\right)^{2}=2 y \sin ^{2} t
$$

so

$$
\gamma^{\prime}(r) \frac{\partial^{2} r}{\partial t^{2}} \leq-2 y \sin ^{2} t \quad \text { and } \quad \gamma^{\prime}(s) \frac{\partial^{2} s}{\partial t^{2}} \leq-2 y \cos ^{2} t
$$


and it follows that

$$
\gamma^{\prime}(r)\left(\frac{\partial^{2} r}{\partial t^{2}}+\frac{\partial^{2} s}{\partial t^{2}}\right) \leq-2 y<0
$$

for $\pi / 4<t<\pi / 2$.

Going back to $\phi$, we have

$$
\tan \phi=\frac{\gamma^{\prime}(s)}{\gamma^{\prime}(r)} \tan t
$$

Now if we let $m(\rho)=\gamma^{\prime}(\rho)^{2} / \gamma(\rho), 0<\rho<b$, then by $(11), m^{\prime}(\rho) \geq 0$ and it follows that $m(s) \leq m(r)$. Hence

$$
\tan \phi=\frac{\sqrt{m(s) \gamma(s)}}{\sqrt{m(r) \gamma(r)}} \tan t \leq \frac{\sqrt{\gamma(s)}}{\sqrt{\gamma(r)}} \tan t=\cot t \tan t=1,
$$

and hence $0<\phi \leq \pi / 4$. Thus

$$
\begin{aligned}
\left|\frac{\partial r}{\partial t}+\frac{\partial s}{\partial t}\right| & \geq 2 y \sqrt{\frac{\cos ^{2} t}{\gamma^{\prime}(s)^{2}}+\frac{\sin ^{2} t}{\gamma^{\prime}(r)^{2}} \sin \left(t-\frac{\pi}{4}\right)} \\
& =2 y \sqrt{\frac{\cos ^{2} t}{(y \sin 2 t)^{2}}\left(\frac{\partial s}{\partial t}\right)^{2}+\frac{\sin ^{2} t}{(y \sin 2 t)^{2}}\left(\frac{\partial r}{\partial t}\right)^{2}} \sin \left(t-\frac{\pi}{4}\right) \\
& =\sqrt{\frac{1}{\sin ^{2} t}\left(\frac{\partial s}{\partial t}\right)^{2}+\frac{1}{\cos ^{2} t}\left(\frac{\partial r}{\partial t}\right)^{2}} \sin \left(t-\frac{\pi}{4}\right) \\
& \geq \sqrt{\left(\frac{\partial r}{\partial t}\right)^{2}+\left(\frac{\partial s}{\partial t}\right)^{2}} \sin \left(t-\frac{\pi}{4}\right) \\
& \geq \sqrt{2 \frac{\partial r}{\partial t}\left|\frac{\partial s}{\partial t}\right|\left(\frac{2}{\pi}\right)\left(t-\frac{\pi}{4}\right)}
\end{aligned}
$$

for $\pi / 4<t<\pi / 2$. Thus

$$
\frac{\sqrt{\frac{\partial r}{\partial t}\left|\frac{\partial s}{\partial t}\right|}}{\left|\frac{\partial r}{\partial t}+\frac{\partial s}{\partial t}\right|}<\frac{\pi}{2 \sqrt{2}} \frac{1}{t-\frac{\pi}{4}}
$$

for $\pi / 4<t<\pi / 2$.

As we saw above, $\partial r / \partial t+\partial s / \partial t$ is negative on the interval $(\pi / 4, \pi / 2)$. Also by (12),

$$
\frac{\partial^{2} r}{\partial t^{2}}+\frac{\partial^{2} s}{\partial t^{2}}<0
$$


so $\partial r / \partial t+\partial s / \partial t$, as a function of $t$, is decreasing on $(\pi / 4, \pi / 2)$, and so $|\partial r / \partial t+\partial s / \partial t|$ is increasing there. Now applying the mean value theorem, we obtain

$$
r+s-r(\tau(y), y)-s(\tau(y), y) \geq(\tau(y)-t)\left|\frac{\partial r}{\partial t}+\frac{\partial s}{\partial t}\right|
$$

for $\pi / 4<t<\tau(y)$. Thus

$$
\begin{aligned}
\frac{\left(\frac{\partial r}{\partial t}\left|\frac{\partial s}{\partial t}\right|\right)^{\frac{1}{4}}}{\sqrt{r+s-r(\tau(y), y)-s(\tau(y), y)}} & \leq \frac{1}{\sqrt{\tau(y)-t}} \frac{\left(\frac{\partial r}{\partial t}\left|\frac{\partial s}{\partial t}\right|\right)^{\frac{1}{4}}}{\left|\frac{\partial r}{\partial t}+\frac{\partial s}{\partial t}\right|^{\frac{1}{2}}} \\
& <\sqrt{\frac{\pi}{2 \sqrt{2}}} \frac{1}{\sqrt{\tau(y)-t}} \frac{1}{\sqrt{t-\frac{\pi}{4}}}
\end{aligned}
$$

for $\pi / 4<t<\tau(y)$. Thus

$$
\begin{aligned}
\int_{\frac{\pi}{4}}^{\tau(y)} & \frac{\sqrt{2}}{\sqrt{r+s-r(\tau(y), y)-s(\tau(y), y)}}\left(\frac{\partial r}{\partial t}\left|\frac{\partial s}{\partial t}\right|\right)^{\frac{1}{4}} d t \\
& \leq \sqrt{\frac{\pi}{\sqrt{2}}} \int_{\frac{\pi}{4}}^{\tau(y)} \frac{d t}{\sqrt{(\tau(y)-t)\left(t-\frac{\pi}{4}\right)}} \\
& =\frac{\pi^{3 / 2}}{2^{1 / 4}} .
\end{aligned}
$$

Thus

and consequently

$$
\mathrm{II} \leq \frac{\pi^{3 / 2}}{2^{1 / 4}} \int_{2 \gamma\left(\frac{|x|}{2}\right)}^{2 \gamma(b)} h(x, y) d y
$$

$$
\mathrm{I} \leq 2^{7 / 4} \pi^{3 / 2} \int_{B(0,2 b)} \int_{2 \gamma\left(\frac{|x|}{2}\right)}^{2 \gamma(b)} h(x, y) d y d x \leq\left(2^{7 / 6} \pi\right)^{3 / 2}\|h\|_{L^{1}\left(\mathbb{R}^{3}\right)} .
$$

\section{Proof of Theorem 1}

Let $f$ be a continuous function on $\mathbb{R}^{3}$ which is compactly supported in the third variable, and let $\gamma \in \mathcal{C}([0, b))$. It is enough to show that

$$
\|\widehat{f d \sigma}\|_{L^{q}\left(\mathbb{R}^{3}\right)} \leq\left(2^{7 / 6} \pi\right)^{3 /(2 q)}\left\|\left(\frac{\gamma(|\cdot|) \gamma^{\prime \prime}(|\cdot|)}{\gamma^{\prime}(|\cdot|)^{2}}\right)^{\frac{\lambda}{2 q}}\right\|_{L^{p_{0}(B(0, b))}}\|f\|_{L^{p}(d \sigma)}
$$

where $d \sigma=\chi_{E} d \sigma_{\gamma}$ and $E=\left\{\left(x_{1}, x_{2}, x_{3}\right) \in \mathbb{R}^{3}: x_{1}, x_{2} \geq 0\right\}$. If $q=\infty$, then this follows easily from Hölder's inequality. So we may assume 
$q<\infty$. Then the relation $1 / p+2 / q=1-1 / p_{0}$ tells us that $p, p_{0}>1$. Also, since

$$
\|\widehat{f d \sigma}\|_{L^{q}\left(\mathbb{R}^{3}\right)}=\|\widehat{f d \sigma} \widehat{f d \sigma}\|_{L^{q / 2}\left(\mathbb{R}^{3}\right)}^{1 / 2}=\|f \widehat{d \sigma * f} d \sigma\|_{L^{q / 2}\left(\mathbb{R}^{3}\right)}^{1 / 2},
$$

and since $q / 2 \geq 2$, it is enough by the Hausdorff-Young inequality to establish that

$$
\begin{aligned}
& \int h|f| d \sigma *|f| d \sigma \\
& \leq\left(2^{7 / 6} \pi\right)^{3 / q}\left\|\left(\frac{\gamma(|\cdot|) \gamma^{\prime \prime}(|\cdot|)}{\gamma^{\prime}(|\cdot|)^{2}}\right)^{\frac{\lambda}{2 q}}\right\|_{L^{p_{0}}(B(0, b))}^{2}\|f\|_{L^{p}(d \sigma)}^{2}\|h\|_{L^{q / 2}\left(\mathbb{R}^{3}\right)}
\end{aligned}
$$

for any nonnegative Lebesgue measurable function $h$ on $\mathbb{R}^{3}$. But by Hölder's inequality,

$$
\int h|f| d \sigma *|f| d \sigma \leq\|f\|_{L^{p}(d \sigma)}^{2}\|h\|_{L^{p^{\prime}}(d \sigma * d \sigma)},
$$

so we need to have

$$
\|h\|_{L^{p^{\prime}}(d \sigma * d \sigma)} \leq\left(2^{7 / 6} \pi\right)^{3 / q}\left\|\left(\frac{\gamma(|\cdot|) \gamma^{\prime \prime}(|\cdot|)}{\gamma^{\prime}(|\cdot|)^{2}}\right)^{\frac{\lambda}{2 q}}\right\|_{L^{p_{0}}(B(0, b))}^{2}\|h\|_{L^{q / 2}\left(\mathbb{R}^{3}\right)} .
$$

Now this follows from Proposition 1 by writing

$$
\begin{aligned}
& \|h\|_{L^{p^{\prime}}(d \sigma * d \sigma)}^{p^{\prime}} \\
= & \int_{\tilde{B}} \int_{\tilde{B}} h^{p^{\prime}}(x+y, \gamma(|x|)+\gamma(|y|)) M(x)^{\frac{p^{\prime}}{2 q}} M(y)^{\frac{p^{\prime}}{2 q}} \frac{N(x)^{\frac{\lambda p^{\prime}}{2 q}} N(y)^{\frac{\lambda p^{\prime}}{2 q}}}{N(x)^{\frac{\lambda p^{\prime}}{2 q}} N(y)^{\frac{\lambda p^{\prime}}{2 q}}} d x d y,
\end{aligned}
$$

where

$$
M(\cdot)=\frac{\gamma^{\prime}(|\cdot|)^{3-2 \lambda} \gamma^{\prime \prime}(|\cdot|)^{\lambda}}{|\cdot| \gamma(|\cdot|)^{1-\lambda}} \text { and } \quad N(\cdot)=\frac{\gamma(|\cdot|) \gamma^{\prime \prime}(|\cdot|)}{\gamma^{\prime}(|\cdot|)^{2}}
$$


and applying Hölder's inequality to get

$$
\begin{aligned}
\|h\|_{L^{p^{\prime}}(d \sigma * d \sigma)}^{p^{\prime}} & \left(\int_{\tilde{B}} \int_{\tilde{B}} N(x)^{\frac{\lambda r p^{\prime}}{2 q}} N(y)^{\frac{\lambda r p^{\prime}}{2 q}} d x d y\right)^{\frac{1}{r}} \\
& \times\left(\int_{\tilde{B}} \int_{\tilde{B}} h^{q / 2}(x+y, \gamma(|x|)+\gamma(|y|)) \frac{M(x)^{\frac{1}{4}} M(y)^{\frac{1}{4}}}{N(x)^{\frac{\lambda}{4}} N(y)^{\frac{\lambda}{4}}} d x d y\right)^{\frac{2 p^{\prime}}{q}} \\
= & \left(\int_{\tilde{B}} N(x)^{\frac{\lambda p_{0}}{2 q}} d x\right)^{\frac{2}{r}} \\
& \times\left(\int_{\tilde{B}} \int_{\tilde{B}} h^{q / 2}(x+y, \gamma(|x|)+\gamma(|y|))\left(\frac{\gamma^{\prime}(|x|)^{3}}{|x| \gamma(|x|)} \frac{\gamma^{\prime}(|y|)^{3}}{|y| \gamma(|y|)}\right)^{\frac{1}{4}} d x d y\right)^{\frac{2 p^{\prime}}{q}} \\
\leq & \left(\int_{\tilde{B}} N(x)^{\frac{\lambda p_{0}}{2 q}} d x\right)^{\frac{2 p^{\prime}}{p_{0}}}\left(\left(2^{7 / 6} \pi\right)^{3 / 2}\left\|h^{q / 2}\right\| L_{L^{1}\left(\mathbb{R}^{3}\right)}\right)^{\frac{2 p^{\prime}}{q}} \\
\leq & \left(2^{7 / 6} \pi\right)^{3 p^{\prime} / q}\left\|\left(\frac{\gamma(|\cdot|) \gamma^{\prime \prime}(|\cdot|)}{\gamma^{\prime}(|\cdot|)^{2}}\right)^{\frac{\lambda}{2 q}\|\|_{L^{p_{0}}(B(0, b))}^{2 p^{\prime}}}\right\| h \|_{L^{q / 2}\left(\mathbb{R}^{3}\right)},
\end{aligned}
$$

where $r$ is the dual exponent to $q /\left(2 p^{\prime}\right)$ (so that $\left.r p^{\prime}=p_{0}\right)$.

\section{References}

[1] A. Carbery And S. Ziesler, Restriction and decay for flat hypersurfaces, Publ. Mat. 46(2) (2002), 405-434.

[2] A. Iosevich, Fourier transform, $L^{2}$ restriction theorem, and scaling, Boll. Unione Mat. Ital. Sez. B Artic. Ric. Mat. (8) 2(2) (1999), $383-387$.

[3] A. Iosevich And G. Lu, Sharpness results and Knapp's homogeneity argument, Canad. Math. Bull. 43(1) (2000), 63-68.

[4] C. E. Kenig, G. Ponce And L. Vega, Oscillatory integrals and regularity of dispersive equations, Indiana Univ. Math. J. 40(1) (1991), 33-69.

[5] A. M. Li, U. Simon And G. S. ZhaO, "Global affine differential geometry of hypersurfaces", de Gruyter Expositions in Mathematics 11, Walter de Gruyter \& Co., Berlin, 1993. 
[6] E. Lutwak, Extended affine surface area, Adv. Math. 85(1) (1991), 39-68.

[7] D. M. Oberlin, A uniform Fourier restriction theorem for surfaces in $\mathbb{R}^{3}$, Proc. Amer. Math. Soc. 132(4) (2004), 1195-1199 (electronic).

[8] P. SuÖLIN, Fourier multipliers and estimates of the Fourier transform of measures carried by smooth curves in $\mathbb{R}^{2}$, Studia Math. 51 (1974), 169-182.

[9] E. M. STEIN, "Harmonic analysis: real-variable methods, orthogonality, and oscillatory integrals", Princeton Mathematical Series 43, Monographs in Harmonic Analysis III, Princeton University Press, Princeton, NJ, 1993.

[10] P. A. Tomas, A restriction theorem for the Fourier transform, Bull. Amer. Math. Soc. 81 (1975), 477-478.

Department of Mathematics

American University of Beirut

Beirut

Lebanon

E-mail address: farukakh@aub.edu.lb

E-mail address: bshayya@aub.edu.lb

Primera versió rebuda el 2 de febrer de 2005,

darrera versió rebuda el 27 de maig de 2005 . 